\title{
DECISIONES ESTRATÉGICAS EN LAS OPERACIONES
}

ADOLFO G. BOUILLON

Profesor de Gestión de Operaciones

Área Académica de Administración

Facultad de Negocios, UPC

Una de las decisiones estratégicas de vital importancia en las operaciones es la del pronóstico de las ventas y de los ingresos. Estos pronósticos permiten cuantificar la cantidad de recursos que son necesarios para que las operaciones se desarrollen de manera óptima y sin contratiempos. Inclusive puede, en entornos de crecimiento económico lento, impulsar las acciones de inversión en infraestructura y capacidad instalada.

Tomemos como ejemplo la industria del retail, ¿Se está presentando un escenario favorable para el crecimiento en esta industria en el Perú? Se evidencia que el sector retail ha tenido buenos resultados en la primera mitad del año (Gestión, 2015), posicionando al Perú como uno de los países con mayor dinamismo en Latinoamérica en esta materia.

Dado que el sector retail es mayormente uno de comercialización, las principales variables en operaciones están relacionadas con la red de locales, expansión del m2 y gestión de los inventarios en productos que suelen tener una alta rotación.

Algunas empresas en el Perú dan muestra de esta expansión, como Zara y Zara Home, que ingresan a Las Begonias en San Isidro; Paris, que abrirá una nueva tienda en Jockey Plaza; Parque Arauco que ve buenas proyecciones de crecimiento en Talara, entre otras iniciativas (Gestión, 2015).

¿Cómo se generan estos pronósticos? Existen varias herramientas, como los modelos asociativos, en donde se utilizan diferentes variables, como nivel de ingreso, ciclo de vida de la unidad familiar, negocios complementarios a desarrollarse en cada zona. Y también están los modelos de series de tiempo, donde se intenta analizar características como la estacionalidad y la tendencia, a un nivel de mediano y corto plazo.

Un modelo interesante de pronóstico es el que plantean los autores Osadchiy, Gaur y Seshadri (2013), en el que se utilizan indicadores financieros, como la bolsa de valores, y la opinión de expertos, para realizar pronósticos de ventas, a través de procesos estocásticos modelados principalmente por movimientos brownianos ${ }^{1}$ y efectos tipo martingala ${ }^{2}$.

Ellos muestran que los indicadores financieros con desfase de un año pueden realizar una adecuada estimación de las ventas operativas para diferentes industrias del retail, superando a otro tipo de modelos como las series de tiempo de variables exclusivamente internas a la empresa.

El nivel de sofisticación de los modelos dependerá de la orientación de la alta gerencia. Por ejemplo, Long, Xu y Ball (2012) combinan modelos de cadenas de Markov con programación dinámica para administrar eficiente mente la probabilidad que un pedido hecho por un cliente se cancele, y si eso no ocurre, que se pueda entregar a tiempo. Desde una perspectiva más amplia, Schlegel (2014) propone un modelo de Big Data junto con analíticas predictivas para administrar adecuadamente el riesgo en la cadena de suministro. Está claro que la importancia de los modelos de pronósticos

\footnotetext{
${ }^{1}$ Un movimiento browniano es un movimiento aleatorio de ciertas partículas sin dirección definida, y se relaciona este comportamiento con el precio de algunos activos financieros, como las acciones.

${ }^{2}$ Una martingala es un proceso estocástico cuyo valor esperado en un tiempo futuro es igual al valor que tiene el proceso en el tiempo actual.
} 
radica en hacer posible las oportunidades de expansión del negocio, ofreciendo una mayor gama de productos a ser adquiridos por los consumidores; que en el caso peruano, éstos representan a una clase media con cada vez mayores aspiraciones y demanda de productos con mayor valor agregado.

\section{Referencias}

Gestión (14 de Setiembre de 2015). Diez nuevos centros comerciales impulsarán sector comercio próximo año. Gestión. Recuperado de https://gestion.pe/economia/diez-nuevos-centroscomerciales-impulsaran-sector-comercio-proximo-ano-99932-noticia/:

Long, G., Xu, S., \& Ball, M. (2012). Managing an Available-to-Promise Assembly System with Dynamic Short-Term Pseudo-Order Forecast. Management Science, 58(4), 770-790.

Osadchiy, N., Gaur, V., \& Seshadri, S. (2013). Sales Forecasting with Financial Indicators and Experts' Input. Production and Operations Management, 22(5), 1056-1076.

Schlegel, G. L. (2014). Utilizing Big Data and Predictive Analytics to Manage Supply Chain Risk. Journal of Business Forecasting, 33(4), 11-17. 